\title{
Viral benefits: murine norovirus can protect the gut via interferon
}

Neil, J.A. et al. Nat. Microbiol. https://doi.org/10.1038/s41564-019-0470-1 (2019)

Much attention to the gut microbiome has been focused on one particular component of that community: bacteria. Without a doubt, the presence or absence of certain bacterial groups or species can influence both health and disease. But bacteria aren't the only microorganisms that call larger hosts home. "People tend to look at the bacterial members of the microbiome but in fact, there are a significant number of viruses that both humans and mice routinely have," says Jessica Neil, a postdoctoral fellow in Ken Cadwell's lab at New York University School of Medicine studying microbiology and the immune system. Just like bacteria, some viruses do indeed cause disease while others occupy the microbiome without pathological consequences; these are particularly prevalent in the intestines, Neil says.

In the mouse world, one example is murine norovirus (MNV). Mice infected with the single-stranded RNA virus are usually asymptomatic (unlike people felled by the human-infecting norovirus that's known for causing outbreaks of gastroenteritis). There is however evidence that the presence of MNV can exacerbate pathologies in mice in certain cases, such as in models of Crohn's and other inflammatory bowel diseases. But it's not all bad news.

In work published in Nature in 2014, Cadwell first showed that MNV can be a positive: infecting germ-free mice, which often develop with abnormal intestinal morphology, with different strains of MNV could improve some of the animals' structural and immunological deficits. The virus also appeared protective against chemically induced damage and pathogenic Citrobacter rodentium infections. "If you infect germ-free mice with murine norovirus, that induces a number of beneficial changes in the gut that are very similar to commensal bacteria," Neil says. But unlike those beneficial bacteria, the benefits of the virus appeared to be mediated through type-1 interferon (IFN-1), a protein involved in regulating activity of the immune system.

In a new paper published in Nature Microbiology, Neil, Cadwell, and colleagues look more closely at the immune signaling

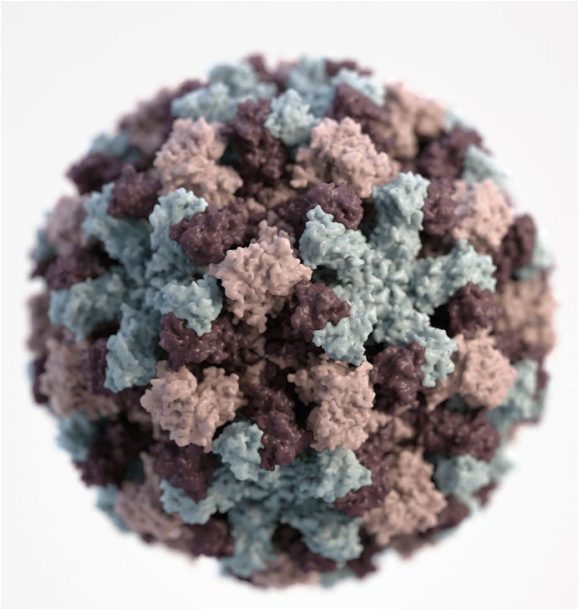

A norovirus particle. Credit: Stocktrek Images, Inc. / Alamy Stock Photo

pathways involved when MNV appears to be playing a protective role in mice.

They first wanted to identify what exactly was special about the most protective of the MNV strains identified previously. With murine norovirus, Neil explains, it's possible to make chimeric versions with mix-andmatched genetic sequences. They were thus able to zero in on one protein produced by the CR6 strain of the virus-nonstructural protein $1 / 2(\mathrm{NS} 1 / 2)$ : in the absence of any other gut microbes, germ-free mice infected with the NS1/2 producing-CR6 strain of MNV had lower mortality after chemical injury to the intestines than virus-free animals or those infected with other MNVs.

To link the protective ability of the CR6 strain to the immune system, the team looked to knock-out mice that lacked immune components that Neil and her colleagues thought most likely to be associated with intestinal repair. Knock out the gene that produces interleukin-22 (IL-22), a cytokine that prompts the proliferation of epithelial cells following damage to the intestines, and the benefits of MNV in germ-free animals were knocked away with it.

Making the link back to IFN-1 took some time, says Neil, but they ultimately identified a pathway that appears to be novel to the virus and involves IFN-1 signaling in the intestinal epithelium. There, the signaling induced by MNV causes populations of monocyte-derived macrophages to accumulate nearby in the intestines; these are dependent on $\mathrm{C}-\mathrm{C}$ chemokine receptor type 2 (CCR2) expression and are involved in prompting another cell type, innate lymphoid cells 3 (ILC3), to in turn kick IL-22 in to gear. This 'monocytes-in-themiddle' sequence, prompted by IFN-1 signaling from epithelial cells, had never been shown before, says Neil.

"What I find exciting about this paper is that we shed light on how an intestinal virus can function like a member of the gut microbiota to be beneficial," commented Cadwell via email. "It turns out that norovirus infection leads to certain immune responses that are similar to beneficial bacteria, but there are many aspects that are completely unique and unexpected. We think that this has important implications for how we view intestinal viruses beyond their role as infectious agents."

The researchers also show an added benefit of MNV during development. In the prior work, viral benefits in adults with intact microbiomes were challenging to clearly tease apart, says Neil, but the microbiomes of developing mice shift as they age. Could MNV be a bigger boost in younger animals, they wondered? So Neil and her colleagues infected three-week old pups with pathogenic C. rodentium: those without MNV CR6 struggled while those with it thrived.

There are still many more details to uncover, says Neil. Are these benefits limited to $\mathrm{MNV}$, or are there other viruses that can influence immune responses? What's going on in the circumstances in which MNV is known to be detrimental, such as in mouse models of Crohn's disease? But whether for better or for worse, there are other members of the microbiome potentially at play in different mouse models. "It's not just the bacterial component that's changing, There are also viruses, and helminths and fungi," says Neil. "Changes in those populations may also be affecting your phenotype."

\section{Ellen P. Neff}

Published online: 15 July 2019

https://doi.org/10.1038/s41684-019-0368-9 